\title{
Professor Dr. Dres. h.c. Adolf Nahrstedt
}

\section{verstorben}

Die Gesellschaft für Phytotherapie trauert um Herrn Professor Dr. Dres. h.c. Adolf Nahrstedt, der am 07. Januar 2016 nach längerer Krankheit verstorben ist.

Adolf Nahrstedt wurde am 09.08.1940 in Northeim (Harz) geboren. Nach dem Vorexamen studierte er an der Universität Freiburg von 1962 bis 1965 Pharmazie und von 1966 bis 1967 Lebensmittelchemie. Im November 1966 wurde er als Apotheker approbiert. Ab 1968 arbeitete Adolf Nahrstedt an seiner Promotion im Fach Pharmakognosie im Arbeitskreis von Prof. Dr. R. Pohl am Pharmakognostischen Institut der Universität Freiburg, die er 1971 mit magna cum laude abschloss. Von 1971 bis 1977 war er als wissenschaftlicher Assistent am Institut für Pharmazeutische Biologie der Universität Freiburg tätig. Im Juni 1976 habilitierte er im Fach Pharmazeutische Biologie und wurde noch im gleichen Jahr auf eine C3 Professur für Pharmazeutische Biologie an die Technische Universität Braunschweig berufen, die er bis 1986 innehatte. Von 1984 bis 1986 arbeitete er als geschäftsführender Leiter des Instituts für Pharmazeutische Biologie an der TU Braunschweig. Im Januar 1986 wurde er auf eine C4 Professur für Pharmazeutische Biologie an die Universität Münster berufen. Prof. Nahrstedt leitete das Institut für Pharmazeutische Biologie als Geschäftsführender Direktor bis September 2004, das während seiner Amtszeit in Institut für Pharmazeutische Biologie und Phytochemie umbenannt wurde.

Im Zentrum seiner wissenschaftlichen Tätigkeit stand die pharmazeutisch-biologische Erforschung von Arzneipflanzen und Naturstoffen. Basis waren stets die chemischen Eigenschaften der Wirkstoffe und der komplexen Extrakte, auf der die

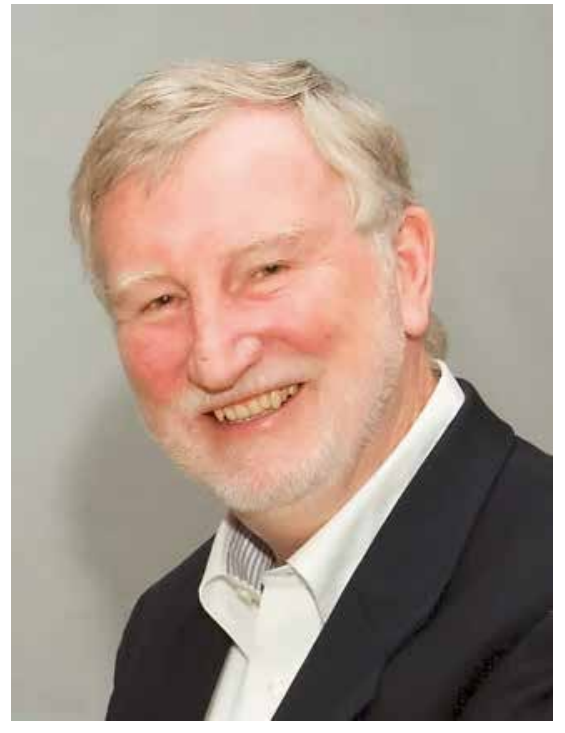

Adolf Nahrstedt (1940-2016)

Wirkungen oder Wirkmechanismen untersucht und diskutiert wurden. Adolf Nahrstedt widmete sich vielfältigen Fragestellungen zur Biochemie und Physiologie pflanzlicher Sekundärmetabolite, insbesondere von Cyanglykosiden. Im Fokus standen zunächst Fragestellungen zur chemischen Ökologie mit dem Hintergrund der Wechselwirkungen zwischen pflanzlichen Naturstoffen und Insekten, später führte er phytochemische und funktionelle Untersuchungen an europäischen Arzneipflanzen durch. Insbesondere interessierte er sich für die Phytochemie von Johanniskrautextrakten sowie die komplexen Interaktionen der Procyanidine und Flavonoide als Koeffektoren zur verbesserten Solubilisierung der Hypericine. Aus diesen Untersuchungen entstanden 250 Originalpublikationen, die bis heute häufig zitiert werden. Über 40 erfolgreich verteidigte Dissertationen zeugen von seiner unermüdlichen Schaf- fenskraft und Ausbildungstätigkeit. Adolf Nahrstedt begleitete zudem einige Nachwuchswissenschaftler zur Habilitation. Auch in der akademischen Selbstverwaltung engagierte er sich. So war er u.a. von 1988 bis 1990 Dekan des Fachbereiches Chemie der Universität Münster, den er während dieser Amtsperiode in den Fachbereich „Chemie und Pharmazie“ umbenennen konnte.

Prof. Nahrstedt engagierte sich auch stark in wissenschaftlichen Fachgesellschaften: Über 9 Jahre war er Vorstandsmitglied in der Gesellschaft für Phytotherapie, von 1981 bis 2005 war er Mitglied des erweiterten Vorstandes der Society of Medicinal Plant and Natural Product Research - Gesellschaft für Arzneipflanzen und Naturstoffforschung (GA). Für die GA übernahm er von 1993 bis 2004 die Schriftleitung der wissenschaftlichen Zeitschrift „Planta Medica“ als Editor in Chief. Unter seiner Leitung wurde „Planta Medica“ zu einem der weltweit führenden Journale im Bereich der Pharmakognosie und Naturstoffwissenschaft. Von 1987 bis 2008 arbeitete er aktiv in der Expertengruppe „Pharmazeutische Biologie“ der Deutschen Arzneibuchkommission mit und war von 2002 bis 2004 stellvertretendes Mitglied der Kommission E beim Bundesinstitut für Arzneimittel und Medizinprodukte.

Am 30. September 2004 trat Adolf Nahrstedt in den Ruhestand. Für seine wissenschaftliche Arbeit wurde er in den folgenden Jahren vielfach gewürdigt und ausgezeichnet: Er erhielt Ehrendoktortitel der Ovidius Universität Constanza, Rumänien sowie der University of Mahasarakham, Mahasarakham in Thailand, die Ehrenmitgliedschaft der Gesellschaft für Arzneipflanzen- und Naturstoffforschung, 


\section{ZPT | forum}

eine Gastprofessur in der Sinai University in Ägypten und ein Adjunct Faculty Membership an der University of Florida at Gainsville, USA. Er wurde zudem mit dem Varro E. Tyler Award der American Society of Pharmacognosy geehrt.

Prof. Adolf Nahrstedt war bei allen Kolleginnen und Kollegen und seinen Kooperationspartnern international beliebt und hoch angesehen. Insbesondere seine analytische, ruhige, besonnene und immer wissenschaftliche Denk- und Arbeitsweise war bemerkenswert. Dabei scheute er den sachlichen wissenschaftlichen Disput zu keiner Zeit.

Die pharmazeutische Wissenschaft und die Phytotherapie verlieren mit Adolf Nahrstedt eine Kapazität und einen wich- tigen Wegbereiter der rationalen Phytotherapie.

Die Gesellschaft für Phytotherapie wird Adolf Nahrstedt stets ein ehrendes Andenken bewahren.

Prof. Dr. Karin Kraft

Präsidentin der Gesellschaft für Phytotherapie

\section{Online}

http://dx.doi.org/10.1055/s-0042-100756 